\title{
Nanodevice for High Precision Readout of Electron Spin
}

\author{
P. Szumniak, S. Bednarek, B. Szafran and P. Grynkiewicz
}

Faculty of Physics and Applied Computer Science, AGH University of Science and Technology al. Mickiewicza 30, 30-059 Kraków, Poland

\begin{abstract}
In this paper we propose and simulate operation of a nanodevice, which enables the electron spin accumulation and very precise read-out of its final value. We exploit the dependence of the electron trajectory on its spin state due to the spin-orbit coupling in order to distinguish between different spin orientations.
\end{abstract}

PACS: 73.21.La, 73.63.Nm, 03.67.Lx, 05.45.Yv

\section{Introduction}

The essential components of a quantum computer are quantum bits (qubits) and an appropriate set of quantum gates. The spin state of electron confined in a semiconductor heterostructure is well suited for physical realization of a quantum bit $[1,2]$. There has been widespread search for nanodevices, which could perform logical operations on such states $[3-5]$. So far the majority of proposed solutions for achieving transitions between different spin states require the application of external microwaves. It is the simplest method of processing information stored in an isolated qubit, but applying it to perform selective operations on single qubit, being part of a many-qubit register is not possible. In order to overcome this problem, we recently proposed the realization of one-qubit logical gates, without the usage of microwaves $[6,7]$.

The main idea of our method is to obtain the spin rotation by the spin-orbit coupling during electron's motion in closed trajectories, which are determined by the metal electrodes deposited on top of a semiconductor heterostructure. The operations are triggered by applying low voltages to the gates. We have simulated operation of the basic one-qubit quantum gates: negation, Hadamard and phase shift $[6,7]$. Two of the problems that remain to be solved are: setting electron's spin in the desired orientation before the logical operation and precise read-out of the quantum computation results.

In this paper we put forward the design of a nanodevice which could serve as an electron spin filter to accumulate electrons in a given spin state in order to set the initial value of qubit and its read-out after quantum computation.

\section{Experimental}

The proposed nanodevice is based on the planar heterostructure described in the previous paper [6]. Its vertical cross-section is depicted in Fig. 1. The nanostruc- ture consists of a quantum well $10 \mathrm{~nm}$ high sandwiched between two tunnel barriers of the same height. The quantum well is separated from strongly doped substrate by $50 \mathrm{~nm}$ thick dopant-free buffer. Metal electrodes are deposited on top of the upper barrier. If one places, in the quantum well, an electron forming a charge cloud, it will induce an opposite charge on the surface of the conductor above. The induced charge attracts the electron and the electric field has a component directed to the center of the cloud. The self-focusing effect of the wave function occurs [8]. As the result the electron is trapped beneath the metal electrode forming a stable wave packet, which exhibits finite spatial extent and conserves its shape as it travels along the path determined by the electrode [9]. Such wave packet shows features unique for a quantum particle to have, as it reflects from a potential barrier or tunnels through it with probability of 1 like a classical object. This fact makes it possible to transfer the electron between different sites of the nanodevice in a controllable manner.

\section{Model}

Despite its classical behavior the electron time evolution is described in the framework of quantum mechanics using time-dependent Schrödinger equation. We also describe electron spin quantum mechanically. The electron is set in motion by voltages applied to the electrodes. We choose the coordinates $x$ and $z$ in the quantum well plane and $y$ pointed in the direction of layer growth. The motion in the $y$ direction is forbidden due to trapping in the quantum well. In the description of the motion in the $(x, z)$ plane we use the following Hamiltonian:

$$
\begin{aligned}
& H(x, z, t)=-\frac{\hbar^{2}}{2 m}\left(\frac{\partial^{2}}{\partial x^{2}}+\frac{\partial^{2}}{\partial z^{2}}\right) \\
& -e \varphi\left(x, y_{0}, z, t\right)+H_{\mathrm{D}},
\end{aligned}
$$

where $y_{0}$ denotes the center of the quantum well, $\varphi(\boldsymbol{r}, t)$ is the electrostatic potential being the result of solving the 
Poisson equation in a box containing entire nanodevice using methods described in our previous papers [5, 8]. The method we use in computation enables us to determine the electron time evolution with the self-focusing effect during its motion. The last term in (1) denotes the Dresselhaus spin-orbit coupling in the quantum well and has the following form:

$$
H_{\mathrm{D}}=\frac{\hbar k_{\mathrm{so}}}{m}\left(p_{x} \sigma_{x}-p_{z} \sigma_{z}\right)
$$

where $\sigma_{x}$ and $\sigma_{z}$ are the Pauli matrices and $k_{\text {so }}$ is the characteristic wave vector dependent on the quantum well width, effective electron mass $m$ and the coupling constant in bulk $\gamma$ :

$$
k_{\mathrm{so}}=\frac{m}{\hbar}\left(\frac{\pi}{d}\right)^{2} \gamma
$$

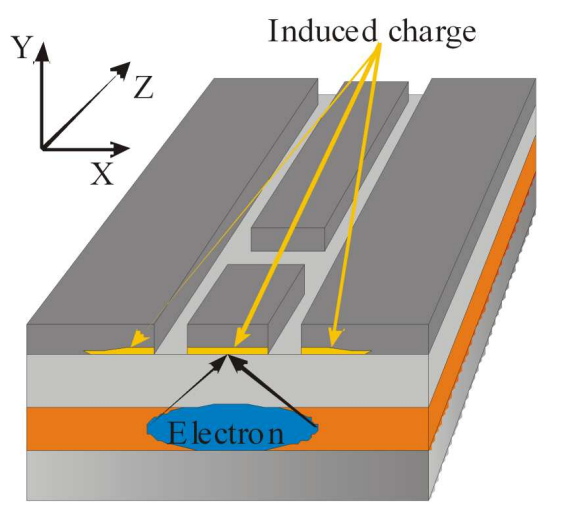

Fig. 1. The layout of the considered nanostructure with electrodes deposited on its surface, wave packet and the induced charge on the lower surface of the electrode.

We denote the wave function as a column matrix

$$
\Psi(x, z, t)=\left(\begin{array}{l}
\psi_{1}(x, z, t) \\
\psi_{2}(x, z, t)
\end{array}\right) .
$$

The simulation described in this paper are the solutions of the time-dependent Schrödinger equation with Hamiltonian (1):

$$
\Psi(t+\mathrm{d} t)=\Psi(t-\mathrm{d} t)-\frac{2 \mathrm{i}}{\hbar} H(t) \Psi(t) \mathrm{d} t .
$$

As the electron charge distribution variates in time, the electrostatic potential $\varphi(\boldsymbol{r}, t)$ has to be computed at each time step. This leads to the time dependence of Hamiltonian (1) introduced in (4). In all simulations the initial wave function is the solution of the time-independent Schrödinger equation:

$$
H(x, z, 0) \Psi(x, z, 0)=E \Psi(x, z, 0)
$$

with potential distribution valid for electron confined beneath particular metal electrode. In our computation we use the material parameters of $\mathrm{ZnTe}$ in which the Dresselhaus coupling constant is $\gamma=13.3 \mathrm{eV} \AA^{3}$, the effective mass $m=0.2 m_{\mathrm{e}}$ and the dielectric constant $\varepsilon=7.4$. Due to large effective mass and small dielectric constant, the self-focusing effect is relatively strong in this material. During electron motion along a straight line the Dresselhaus coupling results in the electron spin rotation around the axis parallel to the movement direction. If it is not forced to move along a straight line, electron trajectory is spin-dependent.

\section{Results and discussion}

Figure 2 depicts trajectories of electrons with different initial spin orientation. The simulation was carried out with the initial wave function obtained for electron trapped beneath the $\mathrm{e}_{1}$ electrode. After starting the iteration of time-dependent Schrödinger equation, the voltage on $\mathrm{e}_{2}$ is increased by $0.1 \mathrm{mV}$, resulting in electron gaining velocity in the $z$ direction. Only the electrons with their spin parallel or anti-parallel to the $z$ axis move along the straight line. In any other case the trajectory drifts in the $x$ direction. Such perturbation of the motion can be used to filter out electrons in undesired spin states.

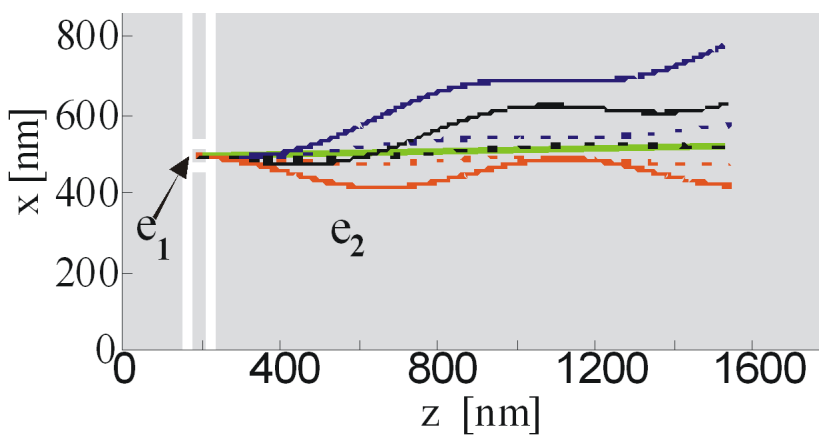

Fig. 2. Trajectories of electrons with diverse initial spin orientation. Continuous lines: blue, red and black for electrons with spin parallel to the axis: $x, y$ and $x y$ (bisector of the angle between $x$ and $y$ axis). Dashed lines for electrons in spin states composed of $90 \%$ of state parallel to the $z$ axis and $10 \%$ of state parallel to axis $x, y$ or $x y$. The straight yellow line stands for electrons with spin precisely parallel or anti-parallel to the $z$ axis (direction of motion).

We put forward the device able to perform such an operation depicted in Fig. 3. Single electron is initially confined beneath $\mathrm{e}_{1}$ electrode. After lowering the voltages on $e_{1}$ and $e_{2}$ the particle is forced to move along $e_{3}$. The width of $e_{3}$ and the distances between $e_{3}$ and the neighboring electrodes are chosen in such a way that the electron cloud centered beneath $\mathrm{e}_{3}$ extends under $\mathrm{e}_{4}$ and $e_{5}$. Then, the slightest lateral perturbation of electron trajectory causes electron to be intercepted by either $\mathrm{e}_{4}$ or $\mathrm{e}_{5}$. Only the electrons with spin parallel or anti-parallel to the $z$ axis are capable of traveling along the entire track beneath $\mathrm{e}_{6}$. One last step of the process remaining is telling between spin up and spin down states. 


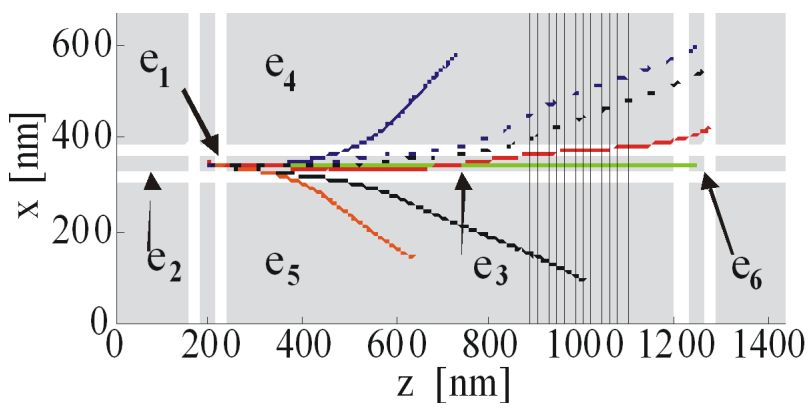

Fig. 3. The layout of electrodes in a nanodevice filtering-out electrons in spin state having any $x$ or $y$ component. The trajectories of electrons with different spins are marked as in Fig. 2. The area marked with vertical lines contains the semimagnetic semiconductor and is used to distinguish between spin parallel to $z$ axis from anti-parallel one.

We propose two possible structures for reaching this goal. First one is based on placing the $e_{3}$ electrode above the quantum well containing semimagnetic semiconductor (an area in which part of $\mathrm{Zn}$ ions is replaced by $\mathrm{Mn}$ ions). If the spins of $\mathrm{Mn}$ ions are polarized by the external magnetic field applied parallel to the $z$ axis, this area is a barrier for electrons with spin anti-parallel to $\mathrm{Mn}$ ion spins.

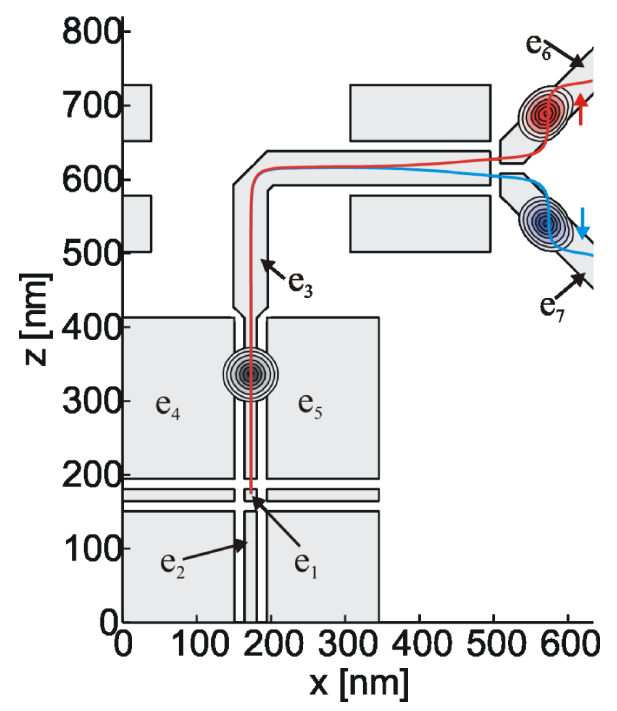

Fig. 4. The nanodevice in which the initial electron spin orientation is measured without applying external magnetic field.

Electrons with spin precisely parallel to Mn ion spins, encounter and cross only a shallow potential cavity, and are the only ones that could reach the $\mathrm{e}_{6}$ electrode. Presented nanodevice can serve to spin accumulation or to spin readout. Unfortunately, in order to polarize Mn ions one has to apply external magnetic field, which is a certain disadvantage, because it leads to persistent spin precession around the direction of the magnetic field.

In alternative structure one manages to measure spin orientation without the necessity of applying external magnetic field. The geometry of this nanodevice and electron trajectories are depicted in Fig. 4. In order to distinguish between spin up and spin down states, we change the direction of electron motion by $90^{\circ}$ forcing it to run in direction of the $x$ axis. The spin-orbit interaction directs the electron in spin-up (spin down) state under $e_{6}\left(e_{7}\right)$ electrode. This nanodevice is able to perform spin read-out. Its main flaw is the destruction of the initial spin state caused by the measurement.

\section{Conclusion}

We put forward and simulated the operating of two alternate nanodevices based on the induced quantum dots and wires, which could be used both to spin accumulation and to perform spin read-out.

\section{Acknowledgments}

This work was supported by the grant No. N N202 128337 from the Ministry of Science and Higher Education, as well as by the "Kraków Interdisciplinary PhD-Project in Nanoscience and Advances Nanostructures" operated within Foundation for Polish Science MPD Programme co-financed by European Regional Development Fund.

\section{References}

[1] D. Loss, D.P. DiVincenzo, Phys. Rev. A 57, 120 (1998).

[2] R. Hanson, L.P. Kouwenhoven, J.R. Petta, S. Tarucha, L.M.K. Vandersypen, Rev. Mod. Phys. 79, 1217 (2007).

[3] J.R. Petta, A.C. Johnson, J.M. Taylor, E.A. Laird, A. Yacoby, M.D. Lukin, C.M. Marcus, M.P. Hanson, A.C. Gossard, Science 309, 2180 (2005).

[4] J.M. Elzermann, R. Hanson, L.H.W. van Beveren, B. Witkamp, L.M.K. Vandersypen, L.P. Kouwenhoven, Nature 430, 431 (2004).

[5] K.C. Nowack, F.H.L. Koppens, Yu.V. Nazarov, L.M.K. Vandersypen, Science 318, 1430 (2007).

[6] S. Bednarek, B. Szafran, Phys. Rev. Lett. 101, 216805 (2008).

[7] S. Bednarek, B. Szafran, Nanotechnology 20, 065402 (2009).

[8] S. Bednarek, B. Szafran, K. Lis, Phys. Rev. B 72, 075319 (2005).

[9] S. Bednarek, B. Szafran, R.J. Dudek, K. Lis, Phys. Rev. Lett. 100, 126805 (2008). 